archives

of thermodynamics

Vol. 36(2015), No. 4, 25-34

DOI: $10.1515 /$ aoter-2015-0030

\title{
Investigation of flow structures during HFE-7100 refrigerant condensation
}

\author{
TADEUSZ BOHDAL ${ }^{a 1}$ \\ MAEGORZATA SIKORA ${ }^{a}$ \\ KATARZYNA WIDOMSKA ${ }^{a}$ \\ ANDRII M. RADCHENKO ${ }^{b}$
}

a Technical University of Koszalin, Department of Heat and Refrigeration Engineering, Racławicka 15-17, 75-620 Koszalin, Poland

$b$ National University of Shipbuilding name after admiral Makarov, Heroev Stalingrada ave. 9, 54025 Nikolaev, Ukraine

\begin{abstract}
The experimental research of environmentally friendly refrigerant HFE-7100 condensation in pipe minichannels was conducted. During the investigations of HFE-7100 condensation in a minichannel with internal diameter $2 \mathrm{~mm}$ together with visualization of flow patterns was made. Visualization results were compared with existing flow structure maps. The identification of the range of flow patterns occurrence during the condensation process of low-pressure refrigerant HFE-7100 was made. The tests were performed throughout the whole range of condensation process.
\end{abstract}

Keywords: Condensation; Minichannels; Flow structures; Refrigerant

\section{Nomenclature}

$d \quad-\quad$ internal diameter, $\mathrm{m}$

$G \quad-\quad$ mass flux density, $\mathrm{kg} /\left(\mathrm{m}^{2} \mathrm{~s}\right)$

$g \quad-$ gravitational acceleration, $\mathrm{m} / \mathrm{s}^{2}$

$J_{v} \quad-$ apparent velocity, $\mathrm{m} / \mathrm{s}$

$\mathrm{T}-$ temperature, ${ }^{\circ} \mathrm{C}$

$x \quad-\quad$ vapour quality,

\footnotetext{
${ }^{1}$ Corresponding Author. E-mail: Tadeusz.Bohdal@tu.koszalin.pl
} 


\section{Greek symbols}

$$
\begin{array}{lll}
\mu & - & \text { kinematic viscosity, Pas } \\
\rho & - & \text { density, } \mathrm{kg} / \mathrm{m}^{3} \\
\chi & - & \text { Lockhart-Martinelli parameter }
\end{array}
$$

\section{Subscripts}

$$
\begin{array}{lll}
h & - & \text { hydraulic } \\
l & - & \text { liquid } \\
s & - & \text { saturation } \\
v & - & \text { vapor }
\end{array}
$$

\section{Introduction}

Due to the development of new technologies leading to the miniaturization of power equipment (while increasing their efficiency), worldwide researches of compact heat exchangers are conducted. Heat exchangers of such type are made of minichannels, which increase the intensity of heat exchange in a small area. The minichannels group are eligible channels with hydraulic diameter $d_{h}<3 \mathrm{~mm}$, in which the flow can be realised (as in conventional channels) in adiabatic conditions (without heat exchange) or nonadiabatic (with heat exchange). Particularly important are nonadiabatic two-phase flows, with the condensation or boiling phase transition.

During the two-phase flow in non-adiabatic conditions different structures than those in conventional channels may occur. Uncritical transferring of the test results of two-phase flow structures from conventional channels to mini - and microchannels leads sometimes to very incorrect descriptions of thermal and flow phenomena. It is similar, to the case of the two-phase flow structure maps of boiling which are adapted to condensation flow structures in the small diameter channels.

It is known that in the channels with small diameter, significant effect on the momentum and energy transport mechanism have the viscosity and surface tension forces, but less important is the influence of gravity and inertia forces [11]. For example in the papers by Coleman and Garimella $[4,6]$, there can be found that for channels with a hydraulic diameter of over $10 \mathrm{~mm}$, channel size and surface tension have no significant impact on the development of the two-phase flow structures. There have been attempts to adapt flow structures maps for two-phase flow in adiabatic conditions, with respect to the condensation flow. An example can be papers due to Łukaszuk [7,8], where an attempt to use Taitel and Dukler maps to the experimental results of two-phase flow of steam in minichannel with hydraulic 
diameter $d_{h}=2.5 \mathrm{~mm}$ was presented. The author concluded, that to a limited extent the map of Taitel and Dukler gives satisfactory approximation with the experimental results in the area of two-phase flow structure recognition. It was reported that for hydraulic diameters above $5 \mathrm{~mm}$ the recognition of flow structures is slightly better, although there are noticeable shifts between these boundaries. Ewing et al. [5] have studied the structures of adiabatic, water - air mixture flow in the horizontal minichannel with internal diameter of $1.6 \mathrm{~mm}$. Attempts to approach the effect of surface tension on the flow structures and description of condensation in minichannels were undertaken in the paper by Tabatabai and Faghri [9] and Akbar et al. [1]. These authors took into account not only the influence of surface tension, but also the viscosity and degree of wetting of the channel walls by the liquid phase.

Knowledge of the two-phase flow structure has an important role in the selection of a suitable correlation for calculation of the heat transfer coefficient and flow resistance. From a physical point of view, various two-phase flow structures are characterized by the following properties:

- bubbly flow - it is a structure of the gas phase (discontinuous gas phase) distributed as bubbles in a continuous liquid phase;

- plug flow - gas bubbles may reach a size comparable to the diameter of the channel and move mostly in the upper part of its cross-section;

- slug flow - with increasing flow rate, shear stress causes an increase in the range of waves of gas bubbles along the flow in channel;

- intermittent - liquid and gaseous phases are separated, generally smooth phase separation surface, usually occurs at low speeds of the two phases;

- wavy - as the speed of gas phase increases, disruption occurs at the interface of the phase separation resulting by formation of waves traveling along the direction of flow;

- annular - the gas phase have a high velocity in a gas core, and on the channel wall surface is formed a liquid film, the thickness of this film is generally asymmetric with respect to the channel cross-section and has a greater thickness at the bottom of the section. 


\section{Experimental investigations}

\subsection{Object of the experiments}

All correlations for calculation of the heat transfer coefficient and flow resistance provided by different authors are defined and valid for the corresponding structure of two-phase flow. Knowledge about the nature of the two-phase flow structure, it is important to define the limits of the possibility of its occurrence. For this purpose there are two-phase flow structure maps. It is known that in the two-phase flow, there is a change in the concentration of phases in the time and space of probabilistic nature, which is very difficult to recognize.

Recognition of the transport mechanism of energy and momentum in the two-phase flow, especially in non adiabatic conditions (e.g., during refrigerant condensation), requires the identification of the flow structures and their boundaries. Analysis of the literature in this area showed two ways of flow structures identification:

- 'calculation' identification (mathematical modeling) - by using twophase flow maps prepared by other authors for different refrigerants in various conditions of the condensation process,

- experimental identification - consisting of flow visualization and image processing.

The authors have attempted to identify the two-phase flow structures in conditions of condensation in minichannels using both methods. Due to authors own database of investigation results [2] and other authors (for medium and high pressure refrigerants) the effectiveness of the calculation method can be developed and determined. For the low-pressure refrigerant hydrofluoroether HFE-7100, in the absence of comparative test results in the literature, authors made their own visualization research in this field. The results of both methods are presented.

\subsection{Testing facility}

Schematic diagram of the test stand for thermal - flow and structure visualization researches of two-phase flow during condensation of HFE-7100 refrigerant is shown in Fig. 1. On the schematic diagram there are listed two sections, marked as A and B. 


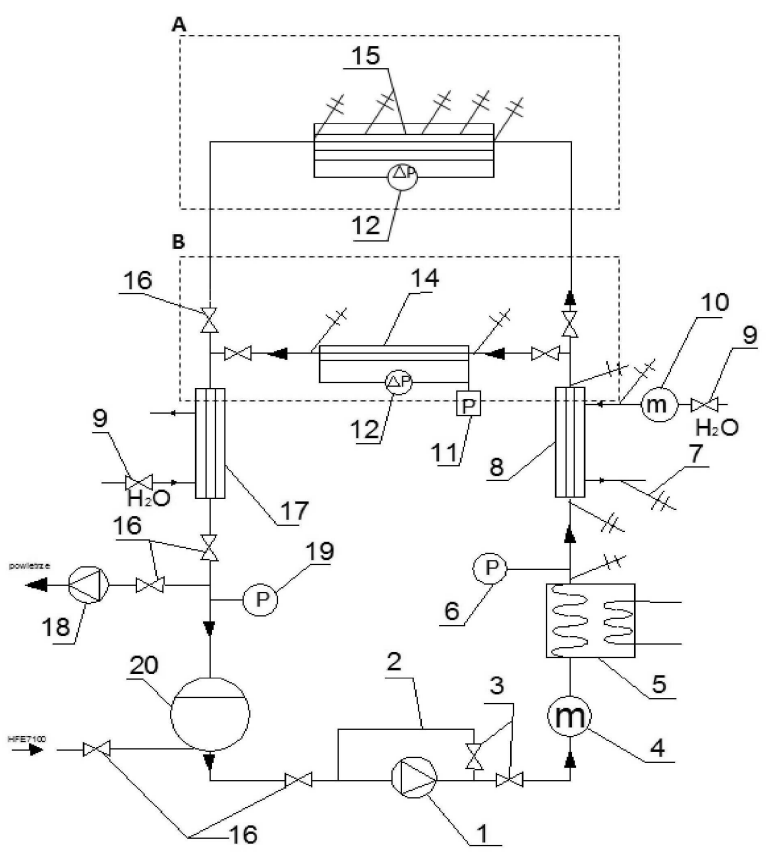

Figure 1: Schematic diagram of the test stand: A - section for thermal and flow investigations, B - section for experimental visualization of two-phase flow structures; 1 - ceramic pump, 2 - bypass for the flow control, 3 - valve, 4 - flowmeter for refrigerant, 5 - evaporator, 6 - manometer, 7 -K-type thermocouples, 8 - pre-heat exchanger 'pipe in pipe' type , 9 - valve, 10 - flowmeter for cooling water, 11 - pressure sensor, 12 - differential pressure sensor, 14 - horizontal section for visualization, 15 - horizontal section of a heat-flow section, 16 - valve, 17 - heat exchanger, 18 - vacuum pump, 19 - manometer, 20 - tank for liquid HFE-7100.

Section A was used for thermal and flow research of HFE-7100 refrigerant condensation in pipe minichannel with internal diameter $d=2 \mathrm{~mm}$. The investigation allowed experimental determination of the average and local values of heat transfer coefficient and flow resistance during condensation. Section B with measurement and recording instrumentation, enables visualization of two-phase flow structures of HFE-7100 refrigerant condensation in minichannel which was formed as a glass tube with a hollow circular channel inside $(d=2 \mathrm{~mm})$. Measurement instrumentation of section B allowed observation of flow structure images under microscope and its recording with the high speed slow motion, high resolution camera, coupled with a computer. Figure 2 shows the overall view of the test stand. 


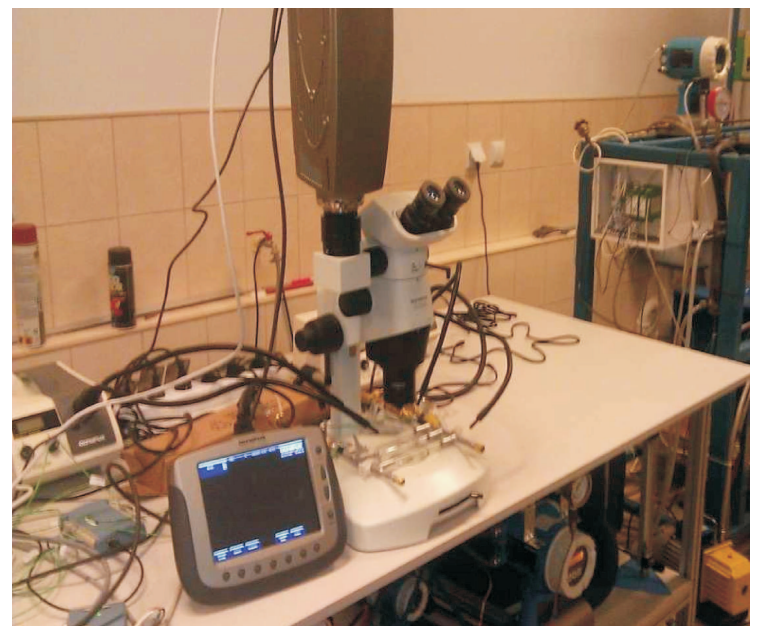

Figure 2: Overall view of the test stand.

In the visualization studies, stereoscopic microscope, with a maximum possible magnification range $4.7 \mathrm{x}$ to $372 \mathrm{x}$ was used. It is designed to observe in transmitted light halogen $150 \mathrm{~W}$ and halogen reflected light with a $2 \times 250 \mathrm{~W}$. High speed camera (i-SPEED type) with CMOS (complementary metal oxide semiconductor) type matrix, with max. recording speed of 10000 fps and max. resolution $1280 \times 1024$ pixels cooperates with the microscope. The camera is equipped with a 4 GB memory. It allows to take measurements of: distance, speed and acceleration. Software of applied camera for PC allows to control, analysis and processing of recordings. Components of visualization equipment are mutually linked with PC. Lighting of the object is allowed by two sets of $250 \mathrm{~W}$ reflected light illuminator. In the visualization study of the two-phase flow structures, during conditions of low-pressure refrigerant HFE-7100, an optical method of image analysis was used.

\section{Results of the investigations}

\subsection{Investigations of two-phase flow structures identifica- tion during condensation in the pipe minichannels}

Using own experimental database, survey investigation results of condensation process of refrigerants: the medium pressure - R134a and high pressure 
- R404A, R407C in pipe minichannels with an internal diameter $d=0.31-$ $3.30 \mathrm{~mm}$, was used 'calculation' method of flow structures identification in condensing conditions. The idea of this method is comparison of experimental results, to the results of calculations according to the criteria used by the other authors structure maps of two-phase flow. This required the determination of the characteristic criteria values with description of the structures boundaries in the terms of experimental research. For the purpose of analysis the apparent velocity of the gas phase, $J_{v}$, and Lockhart-Martinelli parameter, $\chi$, were determined by the relationship:

$$
\begin{gathered}
J_{v}=\frac{G x}{\sqrt{g d \rho_{v}\left(\rho_{l}-\rho_{v}\right)}}, \\
\chi=\left(\frac{1-x}{x}\right)^{0.9}\left(\frac{\rho_{v}}{\rho_{l}}\right)^{0.5}\left(\frac{\mu_{l}}{\mu_{v}}\right)^{0.1} .
\end{gathered}
$$

Consecutive parameters, describing the existence of flow patterns are: vapor quality, $x$, and the mass flux density, $G$. To identify flow structures in experimental results, the flow maps published by authors such as: Thome [10], Cavallini et al. [3], Colleman and Garimella [4] were used (Figs. 3-5). It was found that in the range of parameters, in which was conducted their own experimental studies of refrigerants condensation in minichannels, flow patterns: annular and annular - wavy were observed often, but slug, plug and bubbly patterns were observed rarely. Using two-phase flow structure maps developed by other authors for condensation of tested refrigerants in pipe minichannels was confirmed boundaries of individual structures presence on these maps. Sometimes it is difficult to determine unambiguously the structure which occurs in that moment. In most cases, this type of flow pattern maps have been developed for the R134a refrigerant, which is the best studied refrigerant of chlorofluorocarbon (CFC) substitutes.

\subsection{Investigation results of two - phase flow visualization of HFE-7100 refrigerant in a pipe minichannel}

Experimental study of two-phase flow structures visualization in terms of refrigerant HFE-7100 condensation was carried out on the test stand (Fig. 1) in section $\mathrm{B}$, using pipe minichannel made from glass with an internal diameter $d=2 \mathrm{~mm}$. Visualization of condensation flow structures was made in the top view. Figure 6 shows the description of the image seen in the microscope and recorded with the camera. 


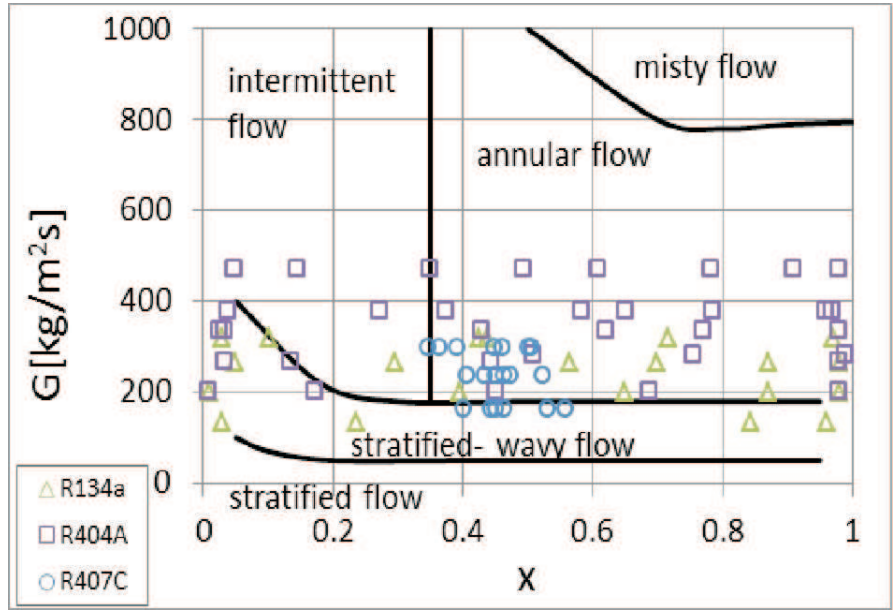

Figure 3: The structure map of two-phase flow by Thome [10] with experimental investiga-tion results of R134a, R404A and R407C refrigerants condensation in minichannel with internal diameter $d=2.3 \mathrm{~mm}$

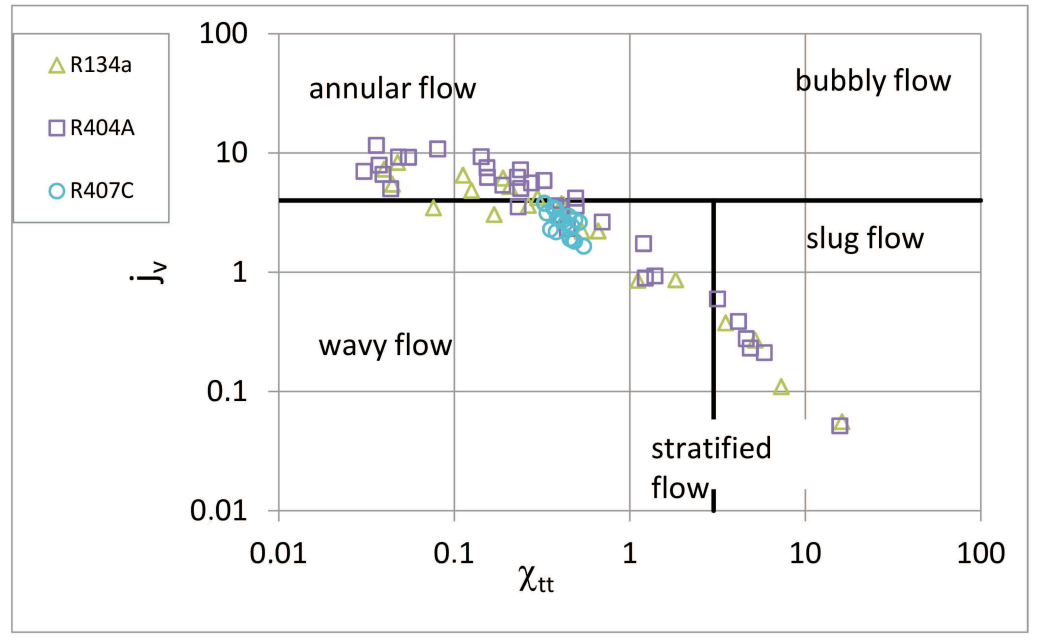

Figure 4: The structure map of two-phase flow by Cavallini [3] with experimental investigation results of R134a, R404A and R407C refrigerants condensation in minichannel with internal diameter $d=2.3 \mathrm{~mm}$ 


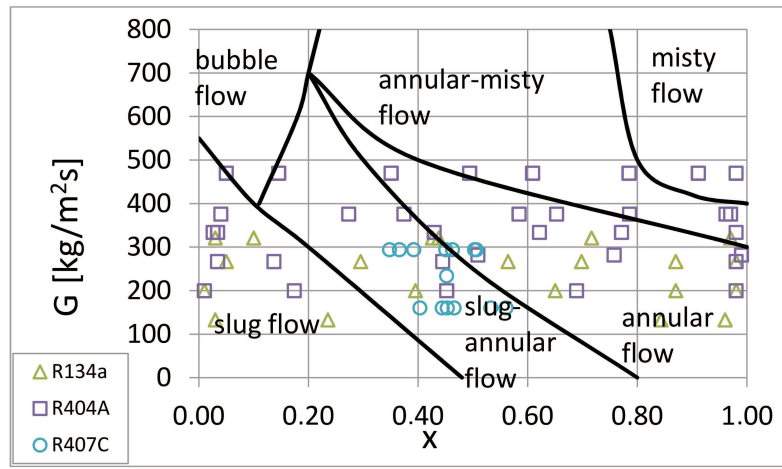

Figure 5: The structure map of two-phase flow by Colleman and Garimella [4] with experimental investigation results of R134a, R404A and R407C refrigerants condensation in minichannel with internal diameter $d=2.3 \mathrm{~mm}$.

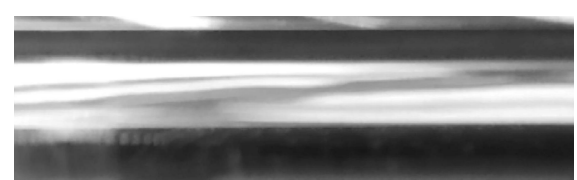

a)

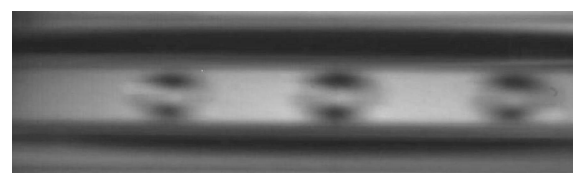

c)

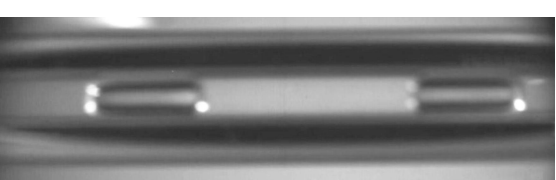

b)

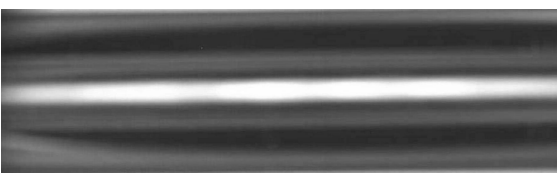

d)

Figure 6: Observed two-phase flow structures during HFE-7100 refrigerant condensation in minichannel with internal diameter $d=2 \mathrm{~mm}$ : a) wavy $-T_{s}=81^{\circ} \mathrm{C}, G=$ $\left.80 \mathrm{~kg} /\left(\mathrm{m}^{2} \mathrm{~s}\right), x=0.62 ; \mathrm{b}\right)$ plug $-T_{s}=83^{\circ} \mathrm{C}, G=56 \mathrm{~kg} /\left(\mathrm{m}^{2} \mathrm{~s}\right), x=0.40$; c) bubbly $\left.-T_{s}=63{ }^{\circ} \mathrm{C}, G=162 \mathrm{~kg} /\left(\mathrm{m}^{2} \mathrm{~s}\right), x=0,30 ; \mathrm{d}\right)$ annular - wavy, $T_{s}=76^{\circ} \mathrm{C}, G=91 \mathrm{~kg} /\left(\mathrm{m}^{2} \mathrm{~s}\right), x=0.69$.

\section{Conclusions}

In the summary of the experimental results of flow structures visualization during HFE-7100 refrigerant condensation the following can be concluded:

1. Low-pressure refrigerant HFE-7100 is useful in modeling of two-phase flow structures during condensation in pipe minichannels.

2. In terms of visualization research in the condensation conditions occurred following structures: plug, wavy, annular - wavy. 
3. With the parameters of condensation in compact minicondensers most often was occurring annular and annular - wave structures.

4. Performed visualization tests showed the possibility of the further implementation of systematic research on two-phase flow structures, that allows to undertake the attempt to generalize two-phase flow structure maps for condensation and not only for HFE-7100 low-pressure refrigerant. In near feature such maps will be developed. This kind of researches can be executed also for other refrigerants.

\section{References}

[1] Akbar M.K., Plummer R.D., Ghiahisian A.: On gas liquid two-phase flow regimes in microchannels. Int. J. Multiphas. Flow 29(2003), 855-865.

[2] Bohdal T., Charun H., Sikora M.: Comparative investigation of the condensation of R134a and R404A refrigerants in pipe minichannels. Int. J. Heat Mass Tran. 54(2011), 1963-1974.

[3] Cavallini A., Del Col D., Doretti L., Matkovic M., Rossetto L., Zilio C.: Two-Phase frictional pressure gradient of R236ea, R134a and R410A inside multi-port mini-channels. Exp. Therm. Fluid Sci. 29(2005), 861-870.

[4] Coleman J., Garimella S.: Two-phase flow regimes in round, square and rectangular tubes during condensation of refrigerant R134a. Int. J. Refrigeration 26(2003), $1,117-128$

[5] Ewing M.E., Weinandy J.J., Christensen R.N.:Observations of two-phase flow patterns in a horizontal circular channel. Heat Transfer Eng. 20(1999), 1, 9-14.

[6] Garimella S.: Condensation flow mechanisms in microchannels: basis for pressure drop and heat transfer models. Heat Transfer Eng. 25(2004), 1, 104-116.

[7] Łukaszuk M.: Application of Taitel and Dukler flow maps for identification of two-phase flow structures in small diameter channels. In: Proc. 19th Nat. Congr. Thermodynamicists Sopot 2005 (in Polish).

[8] ŁukAszuK M.: Experimental investigation of two-phase steam flux in horizontal channels of small diameter. Heat Trans. Eng. 31(2010), 1, 331-334.

[9] Tabatabai A., Faghri A.: A new two-phase flow map and transition boundary accounting for surface tension effects in horizontal miniature and micro tubes. J. Heat Transfer 123(2001), 958-968.

[10] Thome J.R.: Engineering Data Book III: Chapter 8 - Condensation inside tubes. Wolverine Tube, Inc. (2004-2008).

[11] Wengel M., MiŁaszewicz B., Ulbrich R.: Some problems of hydrodynamics of two-phase flow mixtures in minichannels. Arch. Thermodyn. 35(2014), 4, 93-101. 